\title{
Heat and Mass Transfer Effect on Peristalsis of Jeffrey Fluid in a Vertical Channel with Thermal Radiation and Heat Sources
}

\author{
S.Sridhar ${ }^{1}$, V. Ramesh Babu ${ }^{2}$ \\ ${ }^{1}$ Research Scholar, Rayalaseema University, Kurnool, A.P. India \\ ${ }^{2}$ Department Mathematics, Rashtriya Sanskrit Vidyapeetha, Central Deemed University, Tirupati, A.P., India \\ *Corresponding Author:S.Sridhar,Research Scholar, Rayalaseema University, Kurnool, A.P. India, \\ sreedharmsc1@gmail.com
}

\begin{abstract}
We analyse the combined influence of thermal radiation and heat sources on peristaltic flow of a conducting Jeffrey fluid in a vertical porous channel with heat and mass transfer is studied. Using the perturbation technique, the nonlinear governing equations are solved. The expressions for velocity, temperature and concentration the pressure rise per one wave length are determined. The effects of different parameters on the temperature and the pumping characteristics are discussed through graphs.
\end{abstract}

Keywords:Thermal Radiation, Heat sources, Jeffrey fluid, vertical channel, dissipation, peristalsis flow

\section{INTRODUCTION}

Non-Newtonian fluids are referred to those materials that cannot be adequately described by the Navier-Stokes equations. There are materials such as drilling muds, soaps, apple sauce, sugar solution, foams, paste, certain oils, lubricants, clay coating, colloidal and suspension solution, and ketchup, which are now declared as non-Newtonian fluids. Many models in view of diverse properties of such fluids were suggested. For instance, Jamil et al. [14] studied the oscillating flows in a generalized second grade fluid. The unsteady Couette flow of the fractional Maxwell fluid was examined by Athar et al. [4]. Qi and Jin[28] extended such analysis for the generalized Oldroyd-B fluid. Rashidi et al. [30] constructed approximate solutions for the flow and heat transfer in a micropolar fluid. Effects of Soret and Dufour in the magnetohydrodynamic (MHD) flow of the Casson fluid were examined by Hayat et al. [8]. Motsa et al. [24] presented the MHD flow of the upper-convected Maxwell fluid over a porous stretching surface. They used the successive Taylor series linearization method for the solutions of the resulting problem. Effects of MHD and mass transfer of the chemically reactive Maxwell fluid past a porous surface were studied by Vajravelu et al. [35]. It is noticed from the mentioned studies that the rheological parameters in constitutive equations of non-Newtonian fluids make their resulting differential systems more nonlinear and higher order. Such differential systems offer interesting challenges to the researchers from different quarters. The Jeffrey fluid is also one of the models for the non-Newtonian fluids describing the effects of the ratio of relaxation to retardation times and retardation time. Kothandapani and Srinivas [17] used this model for the MHD peristaltic flow of the Jeffrey fluid in an asymmetric channel. Nadeem and Akbar [25] extended the analysis of Ref. [17] for the variable viscosity. Thermal radiation effects in the mixed convection flow of the Jeffrey fluid past a stretching sheet were examined by Hayat et al. [11]. Effects of heat generation/absorption in the Jeffrey fluid flow by a porous stretching sheet were also studied by Hayat et al. [10]. Heat transfer process with radiation effects is very interesting in electrical power generation, solar system technology, space vehicles, missiles, propulsion devices for aircraft, nuclear plants, astrophysical flows, and many other industrial and engineering applications. Although ample studies were generated for the boundary layer flow in the presence of thermal radiation, the fluid thermal conductivity in such cases is treated as a constant. This perhaps is not realistic because it is now proven that the thermal conductivity of liquid metals varies linearly with temperature from $0 \circ \mathrm{F}$ to $400^{\circ} \mathrm{F}$ [Kay [16]]. Thus, the effects of viscous dissipation 
and thermal radiation in the flow of a viscous fluid over a permeable stretching sheet were analysed by Cortell[5]. Pal and Mondal [26] examined the influence of thermal radiation in the hydromagnetic Darcy-Forchheimer mixed convection flow over a stretching surface. Here, the fluid fills the semiinfinite porous space. Combined effects of conduction-radiation in the natural convection flow were addressed by Ashraf et al. [3]. Anbuchezhian et al. [1] examined the thermophoresis and Brownian motion effects in the boundary layer flow of the nanofluid with thermal stratification. Mahmoud and Waheed[22] described the thermal radiation effects in the flow of a micropolar fluid past a permeable plate. Shit and Halder [34] examined the thermal radiation and Hall effects in the MHD flow over an inclined permeable surface. Hayat et al. [11] studied the mixed convection flow of a micropolar fluid with the thermal radiation and chemical reaction. Qasim et al. [27] discussed the thermal radiation in the mixed convection flow of the second-grade fluid over an inclined surface. In all the abovementioned attempts, the flow is considered to be two-dimensional. However, Wang [38] considered the three-dimensional boundary layer flow induced by a stretching surface. In continuation, Ariel [2] developed the homotopy perturbation solution for the flow problem in Ref. [38]. It should be noted that in Refs. [2,38], the flows of viscous fluids without thermal radiation were studied.

The present article discusses the three-dimensional flow of the Jeffrey fluid over a linearly stretching surface when the thermal conductivity varies with temperature. A mathematical model is prepared in the presence of thermal radiation effects. We developed series solutions for the resulting problems by using the homotopy analysis method (HAM) [21]. Results for the velocity, temperature and concentration are constructed. Convergence criteria for the derived series solutions are established. The velocity and temperature are analyzed for various parameters of interest. The local Nusselt number is tabulated and examined. Peristaltic motion in a channel/tube is now known as an important type of flow occurring in several engineering and physiological processes. The peristalsis is well known to the physiologists to be one of the major mechanisms of fluid transport in a biological system and appears in urine transport from kidney to bladder through the ureter, movement of chyme in the gastrointestinal tract, the movement of spermatozoa in the ductus effeerentes of the male reproductive tract and the ovum in the female fallopian tube, the transport of lymph in the lymphatic vessels and vasomotion of small blood vessels such as arterioles, venules and capillaries. Such mechanism has several applications in engineering and in biomedical systems including roller and finger pumps.

The need for peristaltic pumping may arise in circumstances where it is desirable to avoid using any internal moving part such as pistons in pumping process. After the experimental work of Latham [20] on peristaltic transport, Shapiro et al. [32] made a detailed investigation of peristaltic pumping of a Newtonian fluid in a flexible channel and a circular tube. Sud et al. [35] analyzed the pumping action of blood flow in the presence of a magnetic field. Even though it is observed in living systems for many centuries, the mathematical modeling of peristaltic transport began with trend setting works by Shapiro et al. [33] using wave frame of reference and Fung and Yin [39] using laboratory frame of reference.

Hayat et al. [12] studied the peristaltic flow of a micropolar fluid in a channel with different wave frames. Hayat and Ali [7] investigated the peristaltic motion of a Jeffrey fluid under the effect of a magnetic field. Vajravelu et al. [37] studied the peristaltic transport of a Casson fluid in contact with a Newtonian fluid in a circular tube with permeable wall. In physiological peristalsis, the pumping fluid may be considered as a Newtonian or a non-Newtonian fluid. Kapur [15] made theoretical investigations of blood flows by considering blood as a Newtonian as well as non-Newtonian fluids.

Radhakrishnamacharya and Srinivasulu [29] studied the influence of wall properties on peristaltic transport with heat transfer. Mekheimer and Abd Elmaboud [23] analyzed the influence of heat transfer and magnetic field on peristaltic transport of Newtonian fluid in a vertical annulus. Hayat et al. [13] studied the effect of heat transfer on the peristaltic flow of an electrically conducting fluid in a porous space. Krishna Kumari et.al [18] studied the peristaltic pumping of a magnetohydrodynamic casson fluid in an inclined channel. Ravi Kumar et.al [31] considered power-law fluid in the study of peristaltic transport. Krishna Kumari et al [19] has discussed peristaltic pumping conducting Jeffrey fluid in a vertical porous channel with heat transfer. Hayat et al [6] have analysed three-dimensional stretched flow of Jeffrey fluid with variable thermal conductivity and thermal radiation. 
In this paper, peristaltic flow of a conducting Jeffrey fluid in a vertical porous channel with heat and mass transfer is studied. Using the perturbation technique, the nonlinear governing equations are solved. The expressions for velocity, temperature and concentration the pressure rise per one wave length are determined. The effects of different parameters on the temperature and the pumping characteristics are discussed through graphs.

\section{Mathematical Formulation}

We analyse the motion of an electrically conducting, incompressible Jeffrey fluid in a twodimensional vertical porous channel induced by sinusoidal waves propagating with constant speed ' $\mathrm{c}$ ' along the channel walls. For simplicity, we restrict our discussion to the half width of the channel. We assume that a uniform magnetic field of strength Bo is applied normal to the walls as shown in the figure. 1 assuming the magnetic Reynolds to be small we neglect the induced magnetic field.

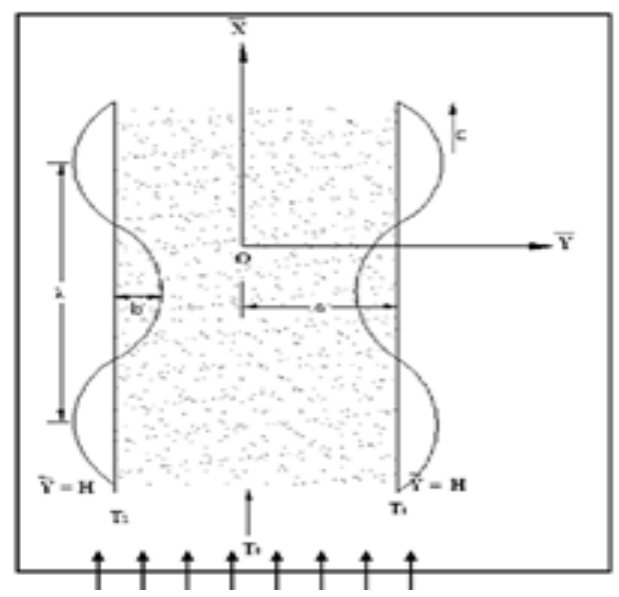

Fig1.Physical model

The wall deformations are given by

$\bar{Y}=H(x, t)=a+b \operatorname{Cos}\left(\frac{2 \pi}{\lambda}(x-c t)\right)($ rightwall $)$

$\bar{Y}=H(x, t)=-a-b \operatorname{Cos}\left(\frac{2 \pi}{\lambda}(x-c t)\right)($ leftwall $)$

where $2 \mathrm{a}$ is the width of the channel, $\mathrm{b}$ is amplitude of the waves and $\lambda$ is the wave length.

The constitutive equations for an incompressible Jeffrey fluid are

$T=-\bar{p} \bar{I}+\bar{s}$

$\bar{s}=\frac{\mu}{1+\lambda_{1}}\left(\overline{\dot{\gamma}}+\lambda_{2} \overline{\dot{\gamma}}\right)$

where $T$ and $s$ are Cauchy stress tensor and extra stress tensor respectively, $p$ is the pressure, $I$ is the identity tensor, $\lambda_{1}$ is the ratio of relaxation to retardation times $\lambda_{2}$ is the retardation time, $\gamma$ is shear rate and dots over the quantities indicate differentiation with respect to time.

In laboratory frame, the continuity equation is

$\frac{\partial \bar{U}}{\partial \bar{X}}+\frac{\partial \bar{U}}{\partial \bar{Y}}=0$

The equations of motion are

$$
\begin{aligned}
& \rho_{0}\left[\bar{U} \frac{\partial \bar{U}}{\partial \bar{X}}+\bar{V} \frac{\partial \bar{U}}{\partial \bar{Y}}\right]=-\frac{\partial \bar{P}}{\partial \bar{X}}+\frac{\partial S_{\bar{x} \bar{x}}}{\partial \bar{X}}+\frac{\partial S_{\bar{x} \bar{y}}}{\partial \bar{X}}-\left(\frac{\mu}{k_{1}}\right) \bar{U}-\left(\sigma \mu_{e}^{2} H_{0}^{2}\right) \bar{U} \\
& +\rho_{o} \beta_{T} g\left(T-T_{o}\right)+\rho_{o} \beta_{C} g\left(C-C_{o}\right) \\
& \rho_{0}\left[\bar{U} \frac{\partial \bar{V}}{\partial \bar{X}}+\bar{V} \frac{\partial \bar{V}}{\partial \bar{Y}}\right]=-\frac{\partial \bar{P}}{\partial \bar{Y}}+\frac{\partial S_{\bar{x} \bar{y}}}{\partial \bar{X}}+\frac{\partial S_{\vec{y} \bar{y}}}{\partial \bar{X}}-\left(\frac{\mu}{k_{1}}\right) \vec{V}
\end{aligned}
$$


The equation of energy is

$$
\begin{gathered}
\rho_{0} C_{p}\left(\bar{U} \frac{\partial T}{\partial \bar{X}}+\bar{V} \frac{\partial T}{\partial \bar{Y}}\right)=k_{f}\left(\frac{\partial^{2} T}{\partial \vec{X}^{2}}+\frac{\partial^{2} T}{\partial \bar{Y}^{2}}\right)-Q_{H}\left(T-T_{o}\right)-\frac{\partial\left(q_{R}\right)}{\partial \bar{Y}} \\
+2 \mu\left(\left(\frac{\partial \bar{U}}{\partial \bar{X}}\right)^{2}+\left(\frac{\partial \vec{V}}{\partial \bar{Y}}\right)^{2}\right)+\mu\left(\frac{\partial \bar{V}}{\partial \bar{X}}+\frac{\partial \bar{U}}{\partial \bar{Y}}\right)+\frac{\mu}{k_{1}}(\bar{U})^{2} \\
\left(\bar{U} \frac{\partial C}{\partial \bar{X}}+\bar{V} \frac{\partial C}{\partial \bar{Y}}\right)=D_{B}\left(\frac{\partial^{2} C}{\partial \vec{X}^{2}}+\frac{\partial^{2} C}{\partial \bar{Y}^{2}}\right)+\frac{D_{T} K_{T}}{T_{m}}\left(\frac{\partial^{2} T}{\partial \vec{X}^{2}}+\frac{\partial^{2} T}{\partial \bar{Y}^{2}}\right)
\end{gathered}
$$

The boundary conditions on, velocity, temperature and Concentration fields are

$$
\begin{aligned}
& \vec{U}=0, T=T_{1}, C=C_{1} \text { at } \bar{Y}=H(\vec{X}) \\
& \frac{\partial \bar{U}}{\partial \bar{Y}}=0, \frac{\partial T}{\partial \bar{Y}}=0 \text { and } \frac{\partial C}{\partial \bar{Y}}=0 \text { at } \bar{Y}=0
\end{aligned}
$$

Where $\vec{U}, \bar{V}$ are the velocity components in the laboratory frame $(\bar{X}, \bar{Y}), \rho_{u}$ is density, $\mu$ is the coefficient of viscosity of the fluid, $c_{p}$ is the specific heat at constant pressure, $\alpha$ is the coefficient of linear thermal expansion of the fluid, $k 0$ is the thermal conductivity, $\mathrm{k}$ is permeability and $\mathrm{T}$ is temperature of the fluid.

The radiation heat term by using The Rosseland approximation is given by

$$
\begin{aligned}
q_{r} & =-\frac{4 \sigma^{\bullet}}{3 \beta_{R}} \frac{\partial T^{\prime 4}}{\partial y} \\
T^{\prime 4} \cong 4 T T 0-3 T_{0}^{4} & \cong \\
\frac{\partial q_{R}}{\partial z} & =-\frac{16 \sigma^{\bullet} T_{0}^{3}}{3 \beta_{R}} \frac{\partial^{2} T}{\partial y^{2}}
\end{aligned}
$$

Using equations $(11-13)$ in equation (8) we get

$$
\begin{aligned}
\rho_{0} C_{p}\left(\bar{U} \frac{\partial T}{\partial \bar{X}}+\bar{V} \frac{\partial T}{\partial \bar{Y}}\right)= & k_{f}\left(\frac{\partial^{2} T}{\partial \vec{X}^{2}}+\frac{\partial^{2} T}{\partial \bar{Y}^{2}}\right)-Q_{H}\left(T-T_{o}\right)+\frac{16 \sigma^{*} T_{0}^{3}}{3 \beta_{R}} \frac{\partial^{2} T}{\partial \bar{Y}^{2}} \\
& +2 \mu\left(\left(\frac{\partial \bar{U}}{\partial \bar{X}}\right)^{2}+\left(\frac{\partial \vec{V}}{\partial \bar{Y}}\right)^{2}\right)+\mu\left(\frac{\partial \bar{V}}{\partial \bar{X}}+\frac{\partial \bar{U}}{\partial \bar{Y}}\right)+\frac{\mu}{k_{1}}(\bar{U})^{2}
\end{aligned}
$$

We shall carryout this investigation in a coordinate system moving with the wave speed $\mathrm{c}$, in which the boundary shape is stationary. The coordinates and velocities in the laboratory frame $(\bar{X} \bar{Y})$ and the wave frame $(\bar{x} \bar{y})$ are related by

$$
\bar{x}=\bar{X}-c t, \bar{y}=\bar{Y}, \bar{u}=\bar{U}-c, \bar{v}=V, \bar{p}=\bar{P}(x, t)
$$

where $\bar{v} \bar{u}$, are the velocity components and $\bar{p}, \bar{P}$, are the pressures in wave and fixed frames. Equations (5)-(9) can be reduced into wave frame as follows

$$
\begin{aligned}
& \frac{\partial \bar{u}}{\partial \bar{x}}+\frac{\partial \bar{v}}{\partial \bar{y}}=0 \\
& \rho_{0}\left[(\bar{u}+c) \frac{\partial \bar{u}}{\partial \bar{x}}+\bar{v} \frac{\partial \bar{u}}{\partial \bar{y}}\right]=-\frac{\partial \bar{p}}{\partial \bar{x}}+\frac{\partial \bar{S}_{\bar{x} \bar{x}}}{\partial \bar{x}}+\frac{\partial \bar{S}_{\bar{x} \bar{y}}}{\partial \bar{y}} \\
& -\left(\sigma \mu_{e}^{2} H_{0}^{2}\right)(\bar{u}+c)-\frac{\mu}{k}(\bar{u}+c)+\rho_{0} g\left(\beta_{T}\left(T-T_{0}\right)+\beta_{C}\left(C-C_{0}\right)\right)
\end{aligned}
$$




$$
\begin{aligned}
& \rho_{0}\left[(\bar{u}+c) \frac{\partial \bar{v}}{\partial \bar{x}}+\bar{v} \frac{\partial \bar{v}}{\partial \bar{y}}\right]=-\frac{\partial \bar{p}}{\partial \bar{x}}+\frac{\partial \bar{S}_{\bar{x} \bar{y}}}{\partial \bar{x}}+\frac{\partial \bar{S}_{\overline{y y}}}{\partial \bar{y}}-\frac{\mu}{k} \bar{v} \\
& \rho_{0} c_{p}\left[(\bar{u}+c) \frac{\partial T}{\partial \bar{x}}+\bar{v} \frac{\partial T}{\partial \bar{y}}\right]=k_{f}\left(\frac{\partial^{2} T}{\partial \bar{x}^{2}}+\frac{\partial^{2} T}{\partial \bar{y}^{2}}\right)-Q_{H}\left(T-T_{o}\right) \\
& +\frac{16 \sigma * T_{0}^{3}}{3 \beta_{R}} \frac{\partial^{2} T}{\partial \bar{Y}^{2}}+2 \mu\left[\left(\frac{\partial \bar{u}}{\partial \bar{x}}\right)^{2}+\left(\frac{\partial \bar{v}}{\partial \bar{y}}\right)^{2}\right]-\left(\frac{\partial \bar{v}}{\partial \bar{x}}+\frac{\partial \bar{u}}{\bar{\partial}}\right)^{2} \frac{\mu}{k}(\bar{u}+c)^{2} \\
& {\left[(\bar{u}+c) \frac{\partial C}{\partial \bar{x}}+\bar{v} \frac{\partial C}{\partial \bar{y}}\right]=D_{B}\left(\frac{\partial^{2} C}{\partial \bar{x}^{2}}+\frac{\partial^{2} C}{\partial \bar{y}^{2}}\right)+\frac{D_{T} K_{T}}{T_{m}}\left(\frac{\partial^{2} T}{\partial \vec{X}^{2}}+\frac{\partial^{2} T}{\partial \bar{Y}^{2}}\right)}
\end{aligned}
$$

Boundary conditions in wave frame are

$$
\begin{aligned}
& \bar{u}+c=0, T=T_{1} \text { and } C=C_{1} \text { at } \bar{y}=H(x) \\
& \frac{\partial \bar{u}}{\partial \bar{y}}=0, \frac{\partial T}{\partial \bar{y}}=0 \text { and } \frac{\partial C}{\partial \bar{y}}=0 \text { at } \bar{y}=0
\end{aligned}
$$

we introduce the following non-dimensional quantities :

$$
\begin{aligned}
& x=\frac{2 \pi \bar{x}}{\lambda}, y=\frac{\bar{y}}{a}, u \frac{\bar{u}}{c}, v=\frac{\bar{v}}{c \delta}, \delta=\frac{2 \pi a}{\lambda}, p \frac{2 \pi a^{2} \bar{p}}{\mu c \lambda}, t=\frac{2 \pi c t^{2}}{\lambda}, \frac{H}{a}, K=\frac{\sqrt{K}}{a} \\
& S=\frac{a}{\mu c} \bar{S}, \varepsilon=\frac{b}{a}, \sigma \frac{a}{\sqrt{k}}, \gamma=\frac{\mu}{\rho_{0}}, T=\theta\left(T_{1}-T_{0}\right)+T_{0}, \\
& C=\phi\left(C_{1}-C_{0}\right)+C_{0}, G r=\frac{\beta_{T}\left(T_{1}-T_{0}\right) a^{3}}{v^{2}}, \\
& \operatorname{Pr} \frac{\mu c_{p}}{k_{0}}, R=\frac{\rho_{0} c a}{\mu}=\frac{c a}{\gamma}, G=\frac{G r}{R}, E c=\frac{c^{2}}{c_{p}\left(T_{1}-T_{0}\right)}, \\
& N=\frac{\beta_{T}\left(C_{1}-C_{0}\right)}{\beta c\left(T_{1}-T_{0}\right)}, R d=\frac{4 \sigma * T_{0}^{3}}{\beta_{R} k_{f}}, M^{2}=\frac{\sigma \mu_{e}^{2} H_{0}^{2}}{}
\end{aligned}
$$

where $\mathrm{R}$ is the Reynolds number, $\delta$ is the dimension less wave number, $\mathrm{K}$ is the permeability parameter, Gr is the Grashof number, Pr is the Prandtl number, $\gamma$ is the Kinematic viscosity of the fluid, Ec is the Eckert number, $\mathrm{Rd}$ is the thermal radiation parameter and $\mathrm{N}$ is buoyancy ratio, $\mathrm{M}$ is the magnetic.

The basic equations (14)-(18) can be expressed in the non-dimensional form as follows

$$
\begin{aligned}
& \frac{\partial u}{\partial x}+\frac{\partial v}{\partial y}=0 \\
& \delta R\left[(u+1) \frac{\partial u}{\partial x}+v \frac{\partial u}{\partial y}\right]=-\frac{\partial p}{\partial x}+\frac{\partial S_{x x}}{\partial x}+\frac{\partial S_{x y}}{\partial y}-\left(\frac{1}{K}+M^{2}\right)(u+1)+G(\theta+N \varphi) \\
& \delta R\left[(u+1) \frac{\partial v}{\partial x}+v \frac{\partial v}{\partial y}\right]=-\frac{\partial p}{\partial x}+\frac{\partial S_{x y}}{\partial x} \delta^{2}+\frac{\partial S_{y y}}{\partial y} \delta-\delta^{2}\left(\frac{1}{K}+M^{2}\right) v \\
& \delta \operatorname{Pr} R\left[(u+1) \frac{\partial \theta}{\partial x}+v \frac{\partial \theta}{\partial y}\right]=\left[\frac{\partial^{2} \theta}{\partial x^{2}} \delta^{2}+\left(1+4 \frac{R d}{3}\right) \frac{\partial^{2} \theta}{\partial y^{2}}\right]+2 \delta^{2} \operatorname{Pr} E c\left[\left(\frac{\partial u}{\partial x}\right)^{2}+\left(\frac{\partial v}{\partial y}\right)^{2}\right] \\
& +\operatorname{Pr} E c\left(\delta^{2} \frac{\partial v}{\partial x}+\frac{\partial u}{\partial y}\right)^{2}+\operatorname{Pr} E c\left(\frac{1}{K}+M^{2}\right)(u+1)^{2}
\end{aligned}
$$


$\delta S c R\left[(u+1) \frac{\partial \varphi}{\partial x}+v \frac{\partial \varphi}{\partial y}\right]=\left[\frac{\partial^{2} \varphi}{\partial x^{2}} \delta^{2}+\frac{\partial^{2} \varphi}{\partial y^{2}}\right]+S c S r\left[\frac{\partial^{2} \theta}{\partial x^{2}} \delta^{2}+\frac{\partial^{2} \theta}{\partial y^{2}}\right]$

where

$S_{x x}=\frac{2 \delta}{1+\lambda_{1}}\left[1+\frac{\delta \lambda_{2} c}{a}\left(u \frac{\partial}{\partial x}+\frac{v}{\delta} \frac{\partial}{\partial y}\right)\right] \frac{\partial u}{\partial x}$

$S_{x y}=\frac{1}{1+\lambda_{1}}\left[1+\frac{\delta \lambda_{2} c}{a}\left(u \frac{\partial}{\partial x}+\frac{v}{\delta} \frac{\partial}{\partial y}\right)\right]\left(\frac{\partial u}{\partial x}+\delta \frac{\partial v}{\partial x}\right)$

$S_{y y}=\frac{-2 \delta}{1+\lambda_{1}}\left[1+\frac{\delta \lambda_{2} c}{a}\left(u \frac{\partial}{\partial x}+\frac{v}{\delta} \frac{\partial}{\partial y}\right)\right] \frac{\partial u}{\partial y}$

and

$\left(\frac{\partial S_{x y}}{\partial y}\right)_{\delta \rightarrow 0}=\frac{1}{1+\lambda_{1}} \frac{\partial^{2} u}{\partial y^{2}}$

The non-dimensional boundary conditions are

$u=-1, \theta=1$ and $\phi=1$ at $y=h$

$\frac{\partial u}{\partial y}, \frac{\partial \theta}{\partial y}$ and $\frac{\partial \phi}{\partial y}=0$ at $y=0$

Using long wave length approximation and dropping terms of order $\delta$ and higher,

It follows equations (21) to (24) are

$$
\begin{aligned}
& 0=-\frac{\partial p}{\partial x}+\frac{1}{1+\lambda_{1}} \frac{\partial^{2} u}{\partial y^{2}}-\left(\frac{1}{K}+M^{2}\right)(u+1)+G(\theta+N \varphi) \\
& 0=-\frac{\partial p}{\partial y} \\
& 0=\left(1+4 \frac{R d}{3}\right) \frac{\partial^{2} \theta}{\partial y^{2}}-\alpha \theta+E c \operatorname{Pr}\left(\frac{\partial u}{\partial y}\right)+E c \operatorname{Pr}\left(\frac{1}{K}+M^{2}\right)(u+1)^{2} \\
& 0=\left[\frac{\partial^{2} \varphi}{\partial x^{2}} \delta^{2}+\frac{\partial^{2} \varphi}{\partial y^{2}}\right]+S c S r\left[\frac{\partial^{2} \theta}{\partial x^{2}} \delta^{2}+\frac{\partial^{2} \theta}{\partial y^{2}}\right] \\
& u=-1, \theta=1 \text { and } \phi=1 \text { at } y=h \\
& \frac{\partial u}{\partial y}, \frac{\partial \theta}{\partial y} \text { and } \frac{\partial \phi}{\partial y}=0 \text { at } y=0
\end{aligned}
$$

The dimensional volume flow rate in the laboratory and wave frames are given by

$$
Q=\int_{0}^{h(\bar{x} t)} \bar{U}(\bar{X}, \bar{Y}, \bar{t}) d \bar{Y}, \quad q=\int_{0}^{h(\bar{x})} \bar{u}(\bar{x}, \bar{y}) d \bar{y}
$$

and now these two are related by the equation

$$
Q=1+c \bar{h}(\bar{x})
$$

The time averaged flow over a period $\mathrm{T}$ at a fixed position $\bar{x}$ is

$\bar{Q}=\frac{1}{T} \int_{0}^{T} Q d t$

\section{Solution Of The Problem}

Equations (31) and (34) are non-linear because they contain three unknowns $\mathrm{u}, \theta$ and $\phi$ which must be solved simultaneously to yield the desired velocity profiles. Due to their nonlinearity they are difficult to solve. However the fact $\mathrm{Ec}$ is small in most practical problems allows us to employ a perturbation technique to solve these non-linear equations. We write

$u=u_{0}+E c u_{1}$ 
$\theta=\theta_{0}+E c \theta_{1}$

$\phi=\phi_{0}+E c \phi_{1}$

Using the above relations, the equations (40), (31)-(34) become

$$
\begin{aligned}
& 0=-\frac{d\left(p_{0}+E c p_{1}\right)}{d x}+\frac{1}{1+\lambda_{1}} \frac{\partial^{2}\left(u_{0}+E c u_{1}\right)}{\partial y^{2}}\left(\frac{1}{K}+M^{2}\right)\left(u_{0}+E c u_{1}+1\right) \\
& +G\left(\left(\theta_{0}+E c \theta_{1}\right)+N\left(\phi_{0}+E c \phi_{1}\right)\right) \\
& 0=\left(1+4 \frac{R d}{3}\right) \frac{\partial^{2}\left(\theta_{0}+E c \theta_{1}\right)}{\partial y^{2}}-\alpha\left(\theta_{0}+E c \theta_{1}\right)+E c \operatorname{Pr}\left[\frac{\partial^{2}\left(u_{0}+E c u_{1}\right)}{\partial y}\right] \\
& +E c \operatorname{Pr}\left(\frac{1}{K}+M^{2}\right)\left(u_{0}+E c u_{1}+1\right)^{2} \\
& 0=\left[\frac{\partial^{2}\left(\phi_{0}+E c \phi_{1}\right)}{\partial y^{2}}\right]+S c \operatorname{Sr}\left[\frac{\partial^{2}\left(\theta_{0}+E c \theta_{1}\right)}{\partial y^{2}}\right] \\
& u_{0}+E c u_{1}=-1 \text { and } \theta_{0}+E c \theta 1=1 \text { at } y=h \\
& \frac{\partial\left(u_{0}+E c\right)}{\partial y}=0, \frac{\partial\left(\theta_{0}+E c \theta_{1}\right)}{\partial y}=0 \text { and } \frac{\partial\left(\phi_{0}+E c \phi_{1}\right)}{\partial y}=0 \text { at } y=0
\end{aligned}
$$

\section{ZEROTH ORDER SOLUTION}

By comparing constant terms on both sides of the above equations we get the zeroth order equations as below

$$
\begin{aligned}
& 0=-\frac{d p_{0}}{d x}+\frac{1}{1+\lambda_{1}} \frac{\partial^{2} u_{0}}{\partial y^{2}}\left(\frac{1}{K}+M^{2}\right)\left(u_{0}+1\right)+G\left(\theta_{0}+N \phi_{0}\right) \\
& 0=\left(1+4 \frac{R d}{3}\right) \frac{\partial^{2} \theta_{0}}{\partial y^{2}}-\operatorname{Pr} \alpha \theta_{0} \\
& 0=\frac{\partial^{2} \phi_{0}}{\partial y^{2}}+S c S r \frac{\partial^{2} \theta_{0}}{\partial y^{2}} \\
& u=-1, \theta=1 \text { and } \phi=1 \text { at } y=h \\
& \frac{\partial u_{0}}{\partial y}=0, \frac{\partial \theta_{0}}{\partial y}=0 \text { and } \frac{\partial \phi_{0}}{\partial y}=0 \text { at } y=0
\end{aligned}
$$

Solving the equations (29) and (30) with the boundary conditions (31), we obtain

$$
\begin{aligned}
& u_{0}=a_{5}\left(\frac{C h\left(\beta_{3} y\right)}{C h \beta_{1} h} C h \beta_{1} h-C h \beta_{1} y\right)+a_{4}\left(1-\frac{C h\left(\beta_{3} y\right)}{C h \beta_{1} h}\right)-\frac{C h \beta_{3} y}{C h \beta_{3} h} \\
& \theta_{0}=\frac{C h\left(\beta_{1} y\right)}{C h \beta_{1} h} \\
& \phi_{0}=1+\frac{\beta_{2}^{2}}{\beta_{1}^{2}}\left(C h \beta_{1} h-C h \beta_{1} y\right)
\end{aligned}
$$

Using the relation (25) we obtain zeroth order dimensionless mean flow in the laboratory and in the wave frame

$$
\begin{aligned}
Q_{0}= & \int_{0}^{h} u_{0} d y=F_{0}+1 \\
& =\frac{d p_{0}}{d x}\left(\frac{\operatorname{Tanh} \beta_{3} h}{\beta_{3}}-h\right)+\left(\frac{a_{5} \operatorname{Cosh}\left(\beta_{1} h\right)-a_{4}-1}{\beta_{3}}\right) \operatorname{Th} \beta_{3} h+a_{4} h-\frac{a_{5}}{\beta_{1}} \operatorname{Sh} \beta_{1} h
\end{aligned}
$$


The pressure gradient is given by

$$
\frac{d p_{0}}{d x}=\frac{\left.Q_{0}+1+\left(1+a_{4}-a_{5} C h \beta_{1} h\right) T h \beta_{3} h-\frac{a_{4} h}{\beta_{3}} \frac{\beta_{3}}{\beta_{1}} a_{5} S h \beta_{1} h\right)}{\left(T h \beta_{3} h-\beta_{3} h\right)}
$$

The non-dimensional zeroth order pressure rise is given by

$$
\Delta p_{0}=\int_{0}^{1} \frac{d p_{0}}{d x} d x
$$

Time mean flow (time averaged flow rate)

$$
\overline{Q_{0}}=\frac{1}{T} \int_{0}^{T} Q_{0} d t=F_{0}+1
$$

where $\beta_{1}^{2}=\frac{3 \alpha R d}{3 R d+4}, \beta_{2}^{2}=\frac{\operatorname{ScSr} \beta_{1}^{2}}{\operatorname{Ch}(\beta, h)}, \beta_{3}^{2}=\mu^{2}\left(1+\lambda_{1}\right), \beta_{4}=\frac{\beta_{1}+\beta_{3}}{2}, \beta_{5}=\frac{\beta_{1}+\beta_{3}}{2} a_{1}=G N\left(\frac{\beta_{2}^{2}}{\beta_{1}^{2}} \operatorname{Ch}(\beta, h)\right), a_{2}=$ $\frac{G}{C g \beta, h}-\frac{G N \beta_{2}^{2}}{\beta_{1}^{2}}, a_{3}=M_{1}^{2}\left(1+\lambda_{1}\right)-a_{1}, a_{4}=\frac{-a_{3}}{M_{1}^{2}\left(1+\lambda_{1}\right)}, a_{5}=\frac{a_{2}}{\beta_{1}^{2}-M_{1}^{2}\left(1+\lambda_{1}\right)}$

\section{First order solution}

From equations (41), (42) and (43) we obtain the first order equations

$$
\begin{aligned}
& 0=-\frac{d p_{1}}{d x}+\frac{1}{1+\lambda_{1}} \frac{\partial^{2} u_{1}}{\partial y^{2}}-\left(\frac{1}{K}+M^{2}\right) u_{1}+G\left(\theta_{1}+N \varphi_{1}\right) \\
& 0=\left(1+4 \frac{R d}{3}\right) \frac{\partial^{2} \theta_{1}}{\partial y^{2}}-\alpha \theta_{1}+\left(\frac{\partial u_{0}}{\partial y}\right)^{2}+\left(\frac{1}{K}+M^{2}\right)\left(u_{0}+1\right)^{2} \\
& 0=\frac{\partial^{2} \phi_{1}}{\partial y^{2}}+S c S r \frac{\partial^{2} \phi_{1}}{\partial y^{2}} \\
& u_{1}=0, \theta_{1}=0 \text { and } \phi_{1}=0 \text { at } y=h \\
& \frac{\partial u_{1}}{\partial y}=0, \frac{\partial \theta_{1}}{\partial y}=0 \text { and } \frac{\partial \phi_{1}}{\partial y}=0 \text { at } y=0
\end{aligned}
$$

Solving the equations (38) and (39) with the use of boundary conditions (40) we obtain

$$
\begin{aligned}
u_{1}=a_{30} & \frac{d p_{1}}{d x}\left(\frac{C h \beta_{1} y}{C h \beta_{1} h}-1\right)+a_{20}\left(\operatorname{Sh} \beta_{1} h \frac{C h \beta_{1} y}{C h \beta_{1} h}-S h \beta_{1} y\right)+a_{23}\left(\frac{C h \beta_{1} y}{C h \beta_{1} h}\right) \\
& +a_{24}\left(C h 2 \beta_{3} h \frac{C h \beta_{1} y}{C h \beta_{1} h}-C h 2 \beta_{3} y\right)+a_{25}\left(C h 2 \beta_{1} h \frac{C h \beta_{1} y}{C h \beta_{1} h}-C h 2 \beta_{1} y\right) \\
& +a_{26}\left(C h \beta_{3} h \frac{C h \beta_{1} y}{C h \beta_{1} h}-C h \beta_{3} y\right)+a_{28}\left(C h \beta_{4} h \frac{C h \beta_{1} y}{C h \beta_{1} h}-C h \beta_{4} y\right) \\
& +a_{29}\left(C h \beta_{5} h \frac{C h \beta_{1} y}{C h \beta_{1} h}-C h \beta_{5} y\right) \\
\theta_{1}=a_{14}( & \left.-\frac{C h \beta_{1} y}{C h \beta_{1} h}\right)+a_{15}\left(C h 2 \beta_{3} h-C h 2 \beta_{3} y \frac{C h \beta_{1} y}{C h \beta_{1} h}\right) \\
+ & a_{11}\left(C h 2 \beta_{1} h-C h 2 \beta_{1} y \frac{C h \beta_{1} y}{C h \beta_{1} h}\right)+a_{17}\left(C h \beta_{3} h-C h \beta_{3} y \frac{C h \beta_{1} y}{C h \beta_{1} h}\right) \\
+ & a_{19}\left(C h \beta_{4} h-C h \beta_{4} y \frac{C h \beta_{1} y}{C h \beta_{1} h}\right)+a_{20}\left(C h \beta_{5} h-C h \beta_{5} y \frac{C h \beta_{1} y}{C h \beta_{1} h}\right)
\end{aligned}
$$




$$
\begin{aligned}
& \phi_{1}=\operatorname{Sc} \operatorname{Sr} \theta_{1} \\
& Q_{1}=F_{1}=\int_{0}^{h} u_{1} d y=\left(\frac{d p_{1}}{d x}\right) a_{31}+\left[a_{32}+a_{33}+a_{34}+a_{35}+a_{36}+a_{37}+a_{38}\right] \\
& =\left(\frac{d p_{1}}{d x}\right) a_{31}+a_{39} \\
& \frac{d p_{1}}{d x}=\frac{Q_{1}-a_{39}}{a_{31}} \\
& \Delta p_{1}=\int_{0}^{1} \frac{d p_{1}}{d x} d x=\int_{0}^{1}\left(\frac{Q_{1}-a_{39}}{a_{31}}\right) d x
\end{aligned}
$$

The friction factor $F$ is given by

$$
F=\int_{0}^{1} h^{2}\left(-\frac{d p_{1}}{d x}\right) d x
$$

The rate of heat transfer $(\mathrm{Z})$ is defined by $Z=\left(\frac{\partial \theta}{\partial y}\right)_{y=h}\left(\frac{\partial y}{\partial x}\right)$

$$
Z=\varepsilon(\operatorname{Sin} x) \beta_{1} T h(\beta h)
$$

Using the relation (25) we obtain first order dimensionless mean flow in the laboratory and in the wave frame

$$
Q_{1}=F_{1}=\int_{0}^{h} u_{1} d y, \quad F_{1}=\frac{d p_{1}}{d x} a_{11}+a_{12}-G a_{1}\left(a_{13}+a_{14}\right)+a_{10} h, \quad \bar{Q}_{1}=\frac{1}{T} \int_{0}^{T} Q_{1} d t=F_{1}
$$

where

$$
\begin{aligned}
& a_{6}=\frac{a_{5} C h \beta_{1} h-a_{4}-1}{C h \beta_{3} h}, a_{7}=\left[M_{1}^{2}\left(\frac{a_{1}^{2}+a_{5}^{2}}{2}+a_{4}^{2}\right)++a_{8}^{2} \beta_{3}^{2}+a_{5}^{2} \beta_{1}^{2}\right], a_{8}=M_{1}^{2} a_{6}^{2}-\frac{a_{6}^{2} \beta_{3}^{2}}{2} \\
& a_{9}=M_{1}^{2} a_{5}^{2}-\frac{a_{5}^{2} \beta_{1}^{2}}{2}, a_{10}=2 \operatorname{Pr} M_{1}^{2} a_{4} a_{6}, a_{11}=2 a_{4} a_{5} \operatorname{Pr} M_{1}^{2}, \\
& a_{12}=\operatorname{Pr}\left[M_{1}^{2}\left(a_{5} a_{6}\right)-2 a_{5} a_{6} \beta_{1} \beta_{3}\right], a_{13}=\operatorname{Pr}\left[-M_{1}^{2} a_{5} a_{6}+2 a_{5} a_{6} \beta_{1} \beta_{3}\right], a_{14}=\frac{a_{7}}{\beta_{1}^{2}}, a_{15}=\frac{a_{8}}{4 \beta_{3}^{2}-\beta_{1}^{2}} \\
& a_{16}=\frac{a_{9}}{3 \beta_{1}^{2}}, a_{17}=\frac{a_{10}}{\beta_{3}^{2}-\beta_{1}^{2}}, a_{18}=\frac{a_{11}}{2 \beta_{1}}, a_{19}=\frac{a_{12}}{\beta_{4}^{2}-\beta_{1}^{2}}, a_{20}=\frac{a_{13}}{\beta_{5}^{2}-\beta_{1}^{2}} \\
& a_{21}=\left(\lambda_{1}+1\right) G(1-S c S r) \frac{\left(-f(h)-a_{18} S c \beta, h\right.}{C h(\beta h)}, a_{22}=\left(\lambda_{1}+1\right) G(1-S c S r) a_{18} \\
& a_{23}=\frac{a_{14}\left(\lambda_{1}+1\right)}{\beta_{1}^{2}} G(1-S c S r), a_{24}=\frac{a_{15}\left(1+\lambda_{1}\right) G(1-S c S r)}{4 \beta_{3}^{2}-\beta_{1}^{2}}, \\
& a_{25}=\frac{a_{16}\left(1+\lambda_{1}\right) G(1-S c S r)}{4 \beta_{1}^{2}}, a_{27}=\frac{a_{18}\left(1+\lambda_{1}\right) G(1-S c S r)}{2 \beta_{1}^{2}}, a_{28}=\frac{a_{19}\left(1+\lambda_{1}\right) G(1-S c S r)}{\beta_{4}^{2}-\beta_{1}^{2}} \\
& a_{29}=\frac{a_{20}\left(1+\lambda_{1}\right) G(1-S c S r)}{\beta_{5}^{2}-\beta_{1}^{2}}, a_{31}=a_{30}\left(\frac{T h \beta h}{h}-h\right), \\
& a_{32}=a_{24}\left(\frac{C h 2 \beta_{3} T h \beta, h}{\beta_{1}}-\frac{S h 2 \beta_{3} h}{2 \beta_{3}}\right), a_{33}=a_{25}\left(\frac{C h 2 \beta, h \operatorname{Th} \beta, h}{\beta_{1}}-\frac{S h 2 \beta, h}{2 \beta_{1}}\right)
\end{aligned}
$$




$$
\begin{aligned}
& a_{34}=a_{26}\left(\frac{\operatorname{Ch} \beta_{3} h T h \beta, h}{\beta_{1}}-\frac{\operatorname{Sh} \beta_{3} h}{\beta_{3}}\right), a_{35}=a_{21}\left(\frac{\operatorname{Th} \beta h}{\beta_{1}}-h\right), a_{36}=a_{23}\left(\frac{\operatorname{Th} \beta h}{\beta_{1}}-h\right) \\
& a_{37}=a_{28}\left(\frac{\operatorname{Ch} \beta_{4} h T h \beta, h}{\beta_{1}}-\frac{\operatorname{Sh} \beta_{4} h}{\beta_{4}}\right), a_{38}=a_{29}\left(\frac{\operatorname{Ch} \beta_{5} h T h \beta, h}{h}-\frac{\operatorname{Sh} \beta_{5} h}{h}\right) \\
& a_{39}=a_{32}+a_{33}+a_{34}+a_{35}+a_{36}+a_{38}
\end{aligned}
$$

The pressure gradient is given by $\frac{d p_{1}}{d x}=\frac{F_{1}-a_{12}+G a_{1}\left(a_{13}+a_{14}\right)+a_{10} h}{a_{11}}=\frac{\bar{Q}_{1}-a_{12}+G a_{1}\left(a_{13}+a_{14}\right)+a_{10} h}{a_{11}}$

The non-dimensional first order pressure rise is given by $\Delta p_{1}=\int_{0}^{1} \frac{d p_{1}}{d x} d x$

The expression for the velocity is given by $u=u_{0}+E c u_{1}$

where $u_{0}$ and $u_{1}$ are given by the equations (32) and (41). The expression for the temperature is obtained as $\theta=\theta_{0}+E c \theta_{1}$ where $\theta_{0}$ and $\theta_{1}$ are given by the equations (33) and (42). The expression for the concentration is obtained as

$\phi=\phi_{0}+E c \phi_{1}$ where $\phi_{0}$ and $\phi_{1}$ are given by the equations (33) and (42). The expression for the pressure rise is $\Delta p=\Delta p_{0}+E c \Delta p_{1}$ where $\Delta p_{0}$ and $\Delta p_{1}$ are given by the equations (36) and (45).

In the absence of mass transfer $(\mathrm{N}=0)$ and heat sources $(\alpha=0)$ the results are good agreement with Krishna Kumari et al [19].

\section{RESULTS AND DISCUSSION}

In this analysis we analyse the effect magnetic field, thermal radiation, dissipation and radiation absorption on peristaltic transport of convective heat and mass transfer flow of an electrically conducting fluid in a symmetric channel in the presence of heat sources. The velocity, temperature, concentration, pressure drop, Friction factor and rate of heat transfer have graphically exhibited for different parametric variations.

Figs.2-7 exhibits the variation of velocity distribution $(\mathrm{u}(\mathrm{y}))$ with different parameters. It can be seen from the profiles that higher the thermal buoyancy force $(\mathrm{G})$ smaller the magnitude of the velocity in the fluid region with maximum attained on the centre $y=0$. Fig. 3 represents the effect of Jeffrey parameter $(\lambda)$. From the profiles we notice an enhancement in $|\mathrm{u}|$ with higher values of $\lambda$. The effect of heat sources on $\mathrm{u}$ is exhibited in fig.4.In the presence of heat generating heat sources, energy is liberated in the flow region, which give to an enhancement in the $|\mathrm{u}|$. Fig.5 show the variation of $\mathrm{u}$ with radiation parameter (Rd). Higher the thermal radiation smaller the magnitude of $u$ with maximum attained on $y=0$. Also, higher the thermo-diffusion $(\mathrm{Sr})$ effects smaller the magnitude of $u$ (fig.6). The effect of buoyancy ratio(N) on $u$ is shown in fig.7.When the molecular buoyancy force dominates over the thermal buoyancy $|\mathrm{u}|$ increases when the forces are in the same directions.

This shows that inclusion of viscous dissipation enhances the magnitude of the velocity in the entire flow region. The temperature distribution $(\theta)$ is exhibited in figs.8-9 for different values of heat source parameter $(\alpha)$ and Thermal radiation parameter $(\mathrm{Rd})$. From fig. 8 we find that in the presence of generating source, heat is generated in the boundary layer, which in turn increase the fluid temperature. It is found that higher the radiative heat flux larger the temperature in the fluid region. It is due to the fact that an increase in $\mathrm{Rd}$, increases the thickness of the thermal boundary layer decreases (fig.9).

The concentration distribution $(\phi)$ with parameters, $\alpha, \mathrm{Rd}$ and $\mathrm{Sr}$ is shown in figs.10-12. From fig.10,12 we notice an increment in the concentration with increasing values of heat generating source, Soret parameter (Sr). This shows that higher the thermo-diffusion effects larger the thickness of the solutal boundary layer. Also, the concentration reduces with increase in thermal radiation parameter (Rd).

The pressure drops $(\Delta \mathrm{P})$ is exhibited in figs.13-18 as a function of $\bar{Q}$ for different values of $\mathrm{G}, \lambda, \alpha, \mathrm{Rd}, \mathrm{Sr}$ and N. An increase in Grashof number $(\mathrm{G})$ reduces the pressure rise. (figs.13). Higher the Jeffrey parameter $(\lambda)$ larger the pressure rise fixing other parameters(fig.14). The variation of $\Delta \mathrm{p}$ with heat source parameter $(\alpha)$ (fig.15) shows that for larger strength of the heat source parameter, we notice a depreciation in $\Delta \mathrm{p}$. Higher radiation parameter/ the thermo-diffusivity effects larger the 
Heat and Mass Transfer Effect on Peristalsis of Jeffrey Fluid in a Vertical Channel with Thermal Radiation and Heat Sources

pressure drop(figs.16\&17). The variation of $\Delta \mathrm{p}$ with buoyancy parameter(N) shows that when the molecular buoyancy force dominates over the thermal buoyancy force, $\Delta \mathrm{p}$ enhances with $\mathrm{N} \leq 1.0$ and reduces with still higher values of $\mathrm{N} \geq 1.5$ (fig.18).

The friction factor (F) as a function of $\vec{Q}$ is depicted in figs, 19-21 for different values of G, $\lambda, \alpha, \mathrm{Rd}, \mathrm{Sr}$ and N. We find from fig.30 that the friction factor reduces with G. Higher Jeffrey fluid parameter $(\lambda)$ /radiation parameter (Rd) smaller Friction factor (fig.20\&22). Lesser the molecular diffusivity/higher thermo-diffusion effects we notice a depreciation in $\mathrm{F}$ (figs.23).Also $\mathrm{F}$ enhances with increase in $\alpha$ (fig.21).With respect to buoyancy $\operatorname{ratio}(\mathrm{N})$ we find that when the molecular buoyancy force dominates over the thermal buoyancy force, $\mathrm{F}$ increases with $\mathrm{N} \leq 1.0$ and reduces with higher $\mathrm{N} \geq$ 1.5 (fig.24).

Figs.25-26 depict the rate of heat transfer(Z) with variations in $\alpha$ and $\mathrm{Rd}$. It can be see from the profiles that rate of heat transfer reduces with increase in heat generating/absorbing source. For higher values of radiation parameter $(\mathrm{Rd})$ we find a depreciation in $\mathrm{Z}$ (fig.26).
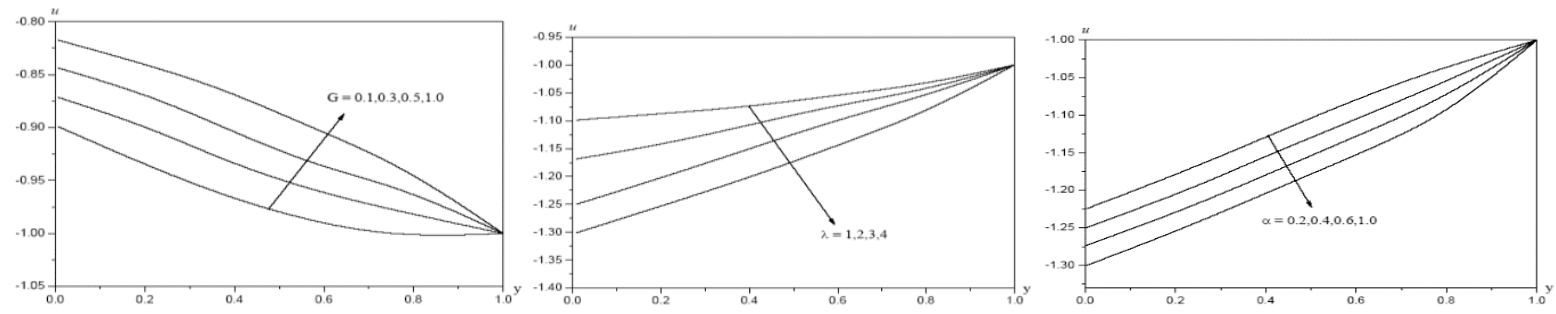

Fig. 2 Effect of $\mathrm{G}$ on $u(\mathrm{y})$ $\lambda=1, \alpha=0.2, \mathrm{Rd}=0.5, \mathrm{Sr}=0.4, \mathrm{~N}=0.5$

Fig. 3 Effect of $\lambda$ on $u(\mathrm{y})$
$\mathrm{G}=0.1, \alpha=0.2, \mathrm{Rd}=0.5, \mathrm{Sr}=0.4, \mathrm{~N}=0.5$

Fig. 4 Effect of $\alpha$ on $u(\mathrm{y})$
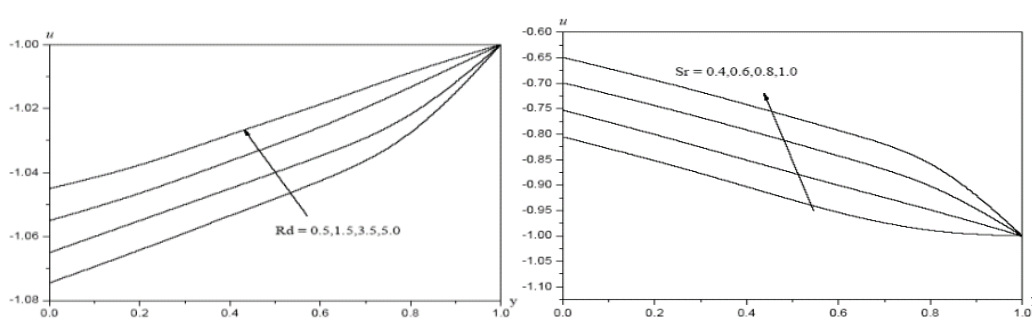

$\mathrm{G}=0,1, \lambda=1, \mathrm{Rd}=0.5, \mathrm{Sr}=0.4, \mathrm{~N}=0.5$

Fig. 5 Effect of Rd on $u$ (y) $\mathrm{G}=0.1, \lambda=1, \alpha=0.2, \mathrm{Sr}=0.4, \mathrm{~N}=0.5$

Fig. 6 Effect of Sr on $u(\mathrm{y})$ $\mathrm{G}=0.1, \lambda=1, \alpha=0.2, \mathrm{Rd}=0.5, \mathrm{~N}=0.5$
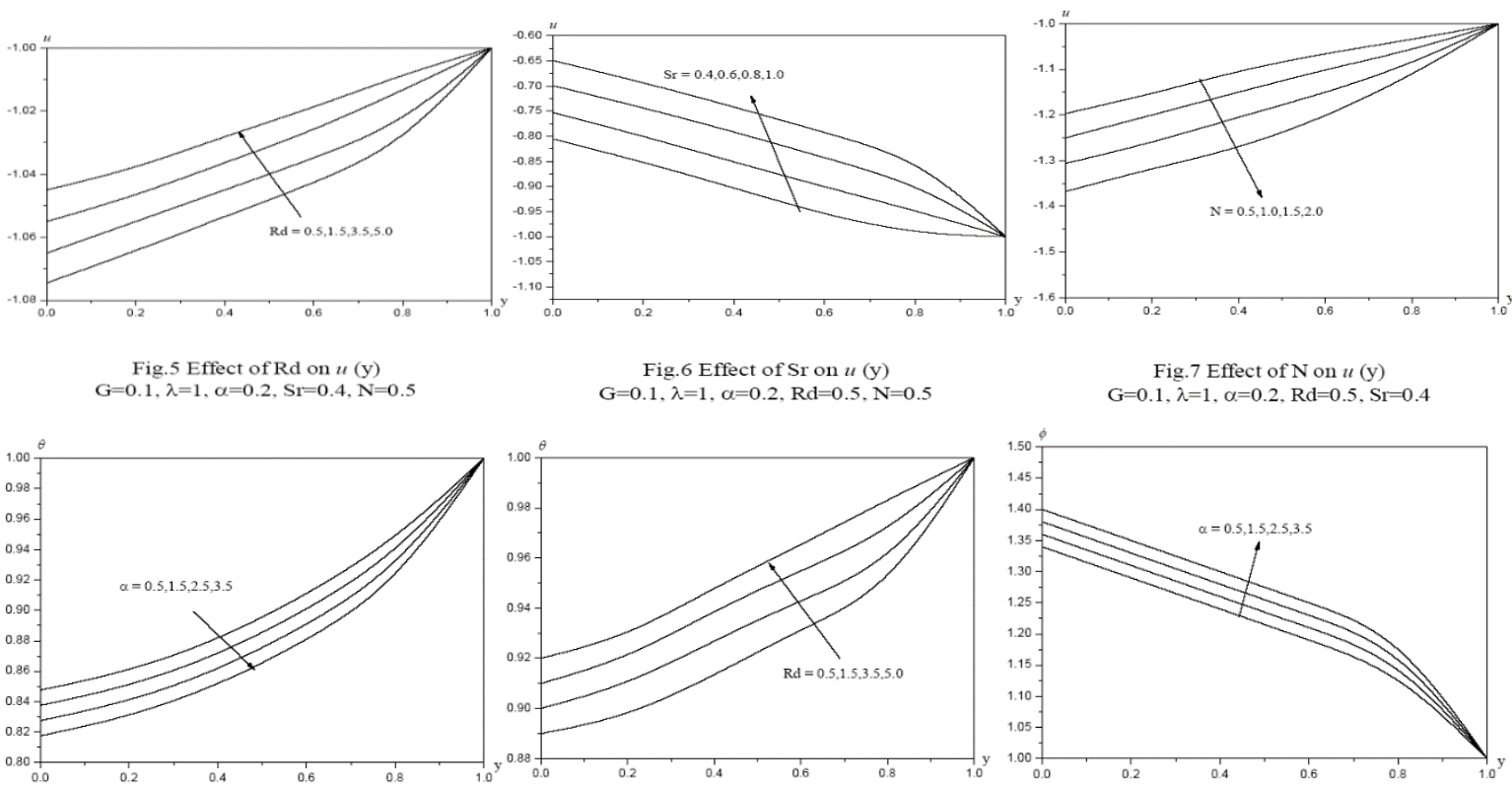

Fig.7 Effect of $\mathrm{N}$ on $u(\mathrm{y})$ Fig. 7 Effect of $\mathrm{N}$ on $u(\mathrm{y})$
$\mathrm{G}=0.1, \lambda=1, \alpha=0.2, \mathrm{Rd}=0.5, \mathrm{Sr}=0.4$

Fig.8 Effect of $\alpha$ on $\theta$ (y) $\mathrm{Rd}=0.5$

Fig.9 Effect of Rd on $\theta$ (y) $\alpha=0.2$

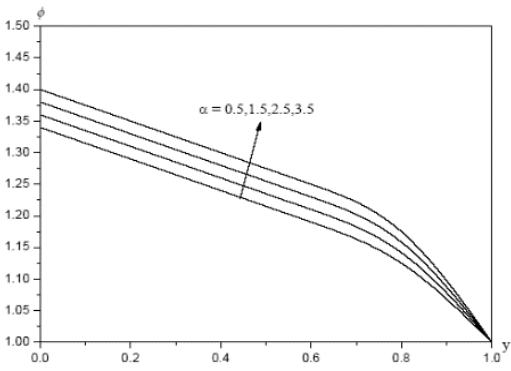

Fig.10 Effect of $\alpha$ on $\phi(y)$ $\mathrm{Rd}=0.5, \mathrm{Sr}=0.4$
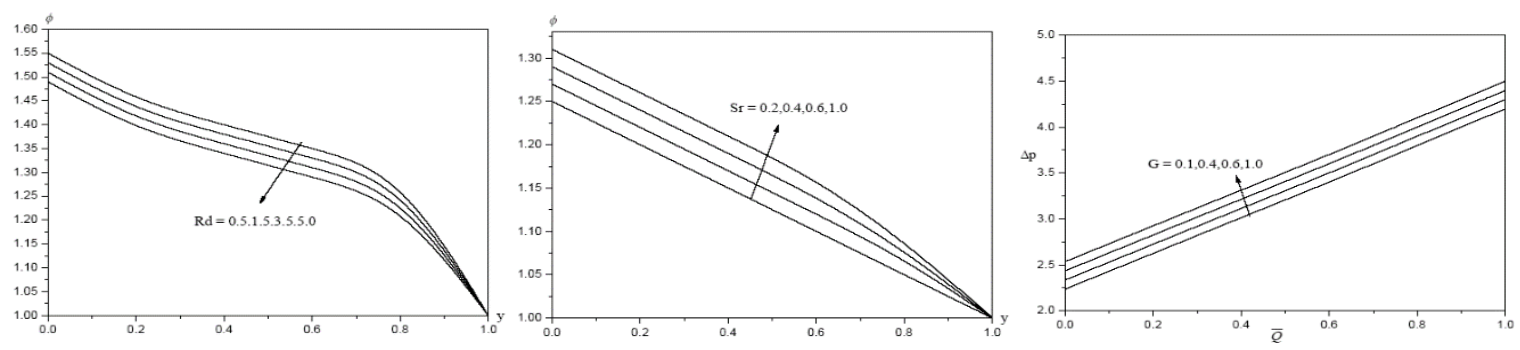

Fig.11 Effect of Rd on $\phi$ (y) $\alpha=0.2 . \mathrm{Sr}=0.4$ 
Heat and Mass Transfer Effect on Peristalsis of Jeffrey Fluid in a Vertical Channel with Thermal Radiation and Heat Sources

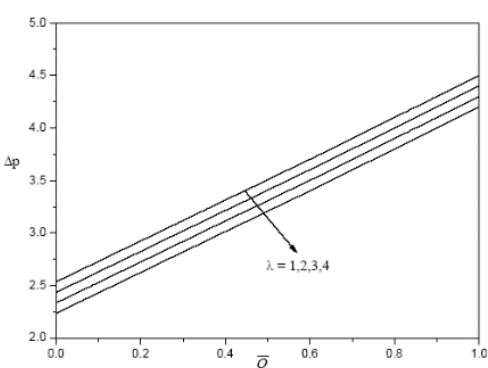

Fig.14 Effect of $\lambda$ on $\Delta \mathrm{p}(\bar{Q})$ $\mathrm{G}=0.1, \alpha=0.2, \mathrm{Rd}=0.5, \mathrm{Sr}=0.4, \mathrm{~N}=0.5$

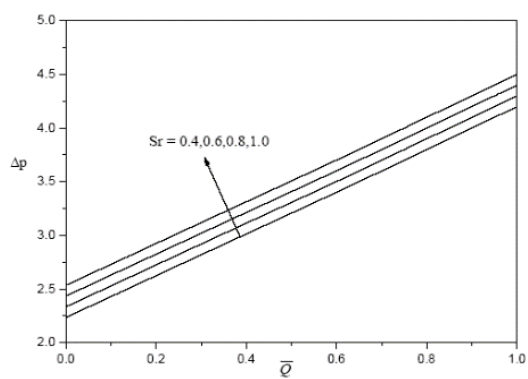

Fig.17 Effect of $\mathrm{Sr}$ on $\Delta \mathrm{p}(\bar{Q})$

$\mathrm{G}=0.1, \lambda=1, \alpha=0.2, \mathrm{Rd}=0.5, \mathrm{~N}=0.5$

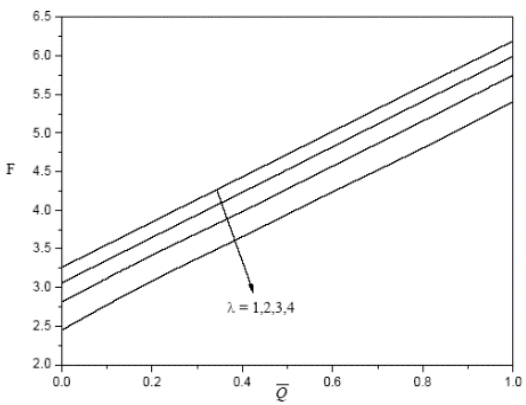

Fig. 20 Effect of $\lambda$ on $\mathrm{F}(\bar{Q})$ $\mathrm{G}=0.1, \alpha=0.2, \mathrm{Rd}=0.5, \mathrm{Sr}=0.4, \mathrm{~N}=0.5$

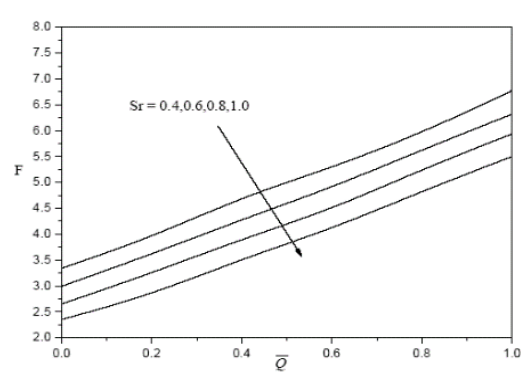

Fig.23 Effect of $\mathrm{Sr}$ on $\mathrm{F}(\bar{Q})$ $\mathrm{G}=0.1, \lambda=1, \alpha=0.2, \mathrm{Rd}=0.5, \mathrm{~N}=0.5$

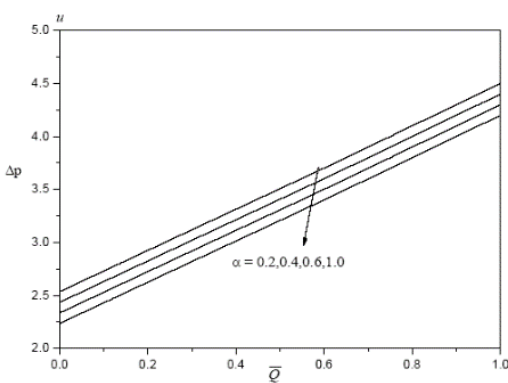

Fig. 15 Effect of $\alpha$ on $\Delta \mathrm{p}(\bar{Q})$ $\mathrm{G}=0.1, \lambda=1, \mathrm{Rd}=0.5, \mathrm{Sr}=0.4, \mathrm{~N}=0.5$

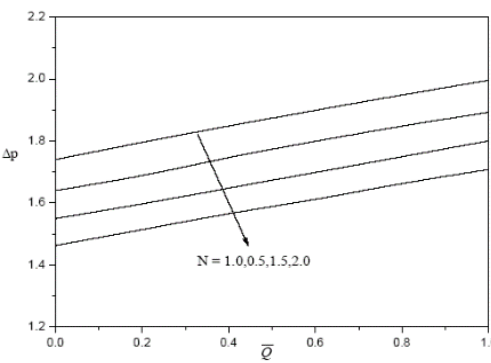

Fig.18 Effect of $\mathrm{N}$ on $\Delta \mathrm{p}(\bar{Q})$ $\mathrm{G}=0.1, \lambda=1, \alpha=0.2, \mathrm{Rd}=0.5, \mathrm{Sr}=0.4$

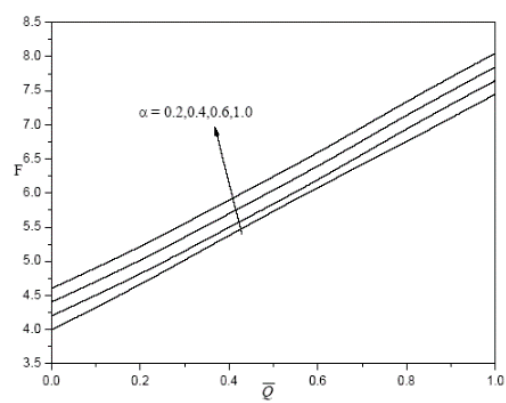

Fig.21 Effect of $\alpha$ on $\mathrm{F}(\bar{Q})$ $\mathrm{G}=0.1, \lambda=1, \mathrm{Rd}=0.5, \mathrm{Sr}=0.4, \mathrm{~N}=0.5$

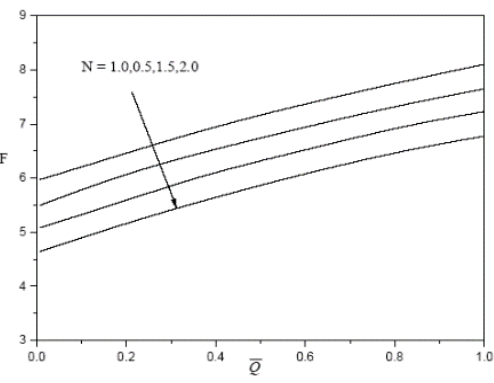

Fig.24 Effect of $\mathrm{N}$ on $\mathrm{F}(\bar{Q})$ $\mathrm{G}=0.1, \lambda=1, \alpha=0.2, \mathrm{Rd}=0.5, \mathrm{~S}=0.4$

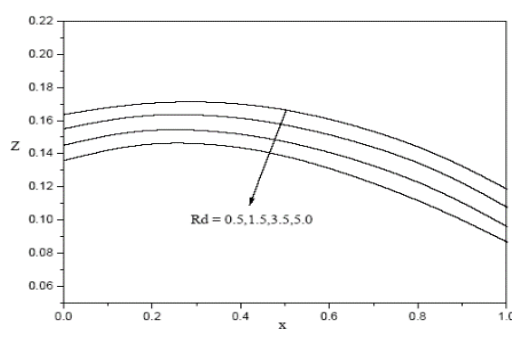

Fig. 26 Effect of Rd on $\mathrm{Z}(\mathrm{x})$ $\alpha=0.2$

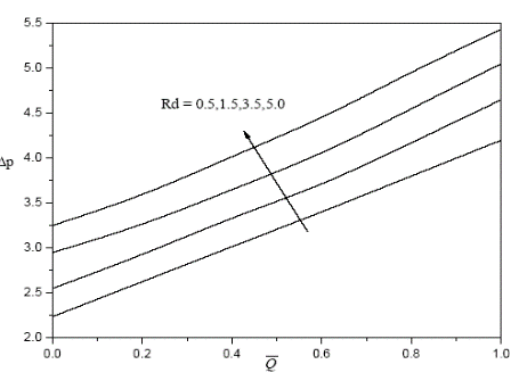

Fig.16 Effect of Rd on $\Delta \mathrm{p}(\bar{Q})$ $\mathrm{G}=0.1, \lambda=1, \alpha=0.2, \mathrm{Sr}=0.4, \mathrm{~N}=0.5$

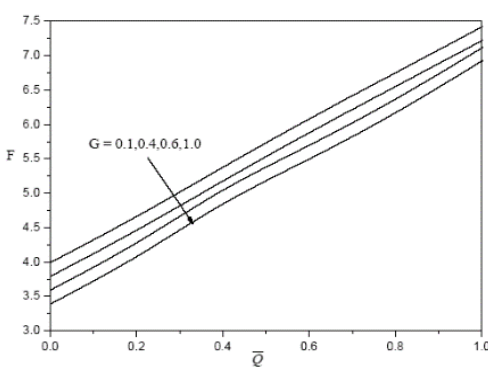

Fig.19 Effect of $\mathrm{G}$ on $\mathrm{F}(\bar{Q})$ $\lambda=1, \alpha=0.2, \mathrm{Rd}=0.5, \mathrm{Sr}=0.4, \mathrm{~N}=0.5$

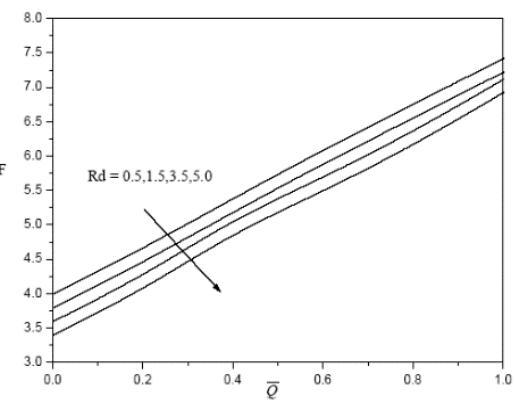

Fig.22 Effect of Rd on $\mathrm{F}(\bar{Q})$ $\mathrm{G}=0.1, \lambda=1, \alpha=0.2, \mathrm{Sr}=0.4, \mathrm{~N}=0.5$

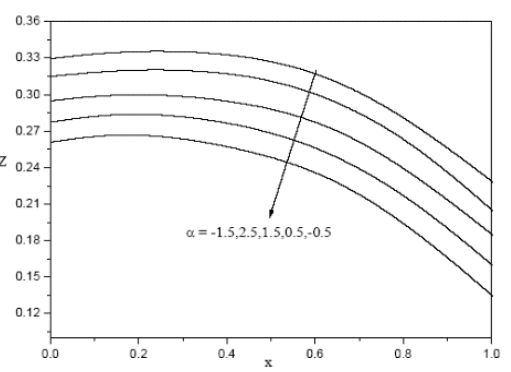

Fig.25 Effect of $\alpha$ on $Z$ (x) $\mathrm{Rd}=0.5$ 
Heat and Mass Transfer Effect on Peristalsis of Jeffrey Fluid in a Vertical Channel with Thermal Radiation and Heat Sources

\section{Conclusions}

The combined influence of thermal radiation and heat sources on convective heat and mass transfer flow of Jeffrey fluid in vertical channel has been discussed for different parametric variations. The important conclusions of this analysis are

- The magnitude of u decreases with G, M, Sc and enhances with $\mathrm{K}$ and $\lambda_{1}$. $|\mathrm{u}|$ enhances with heat sources parameter $\alpha$ and buoyancy ratio $\mathrm{N}$. Higher the thermal radiation and dissipation smaller $|\mathrm{u}|$.

- Higher the strength heat source / thermal radiation / dissipation lager the temperature $(\theta)$ in the flow region.

- The concentration $(\phi)$ enhances with soret parameter $(\mathrm{Sr})$ and heat source parameter $(\alpha)$, reduces with Rd and Ec.

- The presser rise $\Delta \mathrm{p}$ reduces with $\mathrm{G}, \mathrm{M}, \mathrm{K}, \alpha, \mathrm{Sc}$ and enhances with Jeffery parameter $\lambda$ and Ec. $\Delta \mathrm{p}$ enhances with increases with increasing buoyancy ratio $\mathrm{N} \leq 1.0$ and reduces with higher $\mathrm{N} \geq 1.5$.

- The friction factor $(\mathrm{F})$ reduces with $\mathrm{G}, \mathrm{K}, \lambda, \mathrm{Rd}, \mathrm{Sc}, \mathrm{Sr}$ and enhances with magnetic parameter M. The friction factor enhances with increasing buoyancy ratio $\mathrm{N} \leq 1.0$ and reduces with higher $\mathrm{N} \geq$ 1.5 .

- The rate of heat transfer reduces with increasing $\alpha$ and $\mathrm{Rd}$ and enhances with dissipation parameter Ec.

\section{REFERENCES}

[1] Anbuchezhian, N., Srinivasan, K., Chandrasekaran, K., and Kandasamy, R. Thermophoresis and Brownian motion effects on boundary layer flow of nanofluid in presence of thermal stratification due to solar energy. Applied Mathematics and Mechanics (English Edition), 33, 765-780 (2012) DOI 10.1007/s10483012-1585-8

[2] Ariel, P. D. The three-dimensional flow past a stretching sheet and the homotopy perturbation method. Computers and Mathematics with Applications, 54, 920-925 (2007)

[3] Ashraf, M., Asghar, S., and Hossain, M. M. Computational study of combined effects of conductionradiation and hydromagnetics on natural convection flow past magnetized permeable plate. Applied Mathematics and Mechanics (English Edition), 33, 731-748 (2012) DOI 10.1007/s10483-012-1583-7

[4] Athar, M., Fetecau, C., Kamran, M., Sohail, A., and Imran, M. Exact solutions for unsteady axial Couette flow of a fractional Maxwell fluid due to an accelerated shear. Nonlinear Analysis: Modelling and Control, 16, 135-151 (2011)

[5] Cortell, R. Combined effects of viscous dissipation and thermal radiation on fluid flows over a nonlinearly stretched permeable wall. Meccanica, 47, 769-781 (2012)

[6] Hayat T, Shehzad S. A., Alsaed A.: Three-dimensional stretched flow of Jeffrey fluid with variable thermal conductivity and thermal radiation, Appl. Math. Mech. -Engl. Ed., Vol.34(7), pp.823-832 (2013), DOI 10.1007/s10483-013-1710-7.

[7] Hayat, T and Ali, N.,Physica A: Statistical Mechanics and its Applications,2006, 370,225-239.

[8] Hayat, T., Shehzad, S. A., and Alsaedi, A. Soret and Dufour effects on magnetohydrodynamic (MHD) flow of Casson fluid. Applied Mathematics and Mechanics (English Edition), 33, 1301-1312 (2012) DOI 10.1007/s10483-012-1623-6

[9] Hayat, T., Shehzad, S. A., and Qasim, M. Mixed convection flow of a micropolar fluid with radiation and chemical reaction. International Journal for Numerical Methods in Fluids, 67, 1418-1436 (2011)

[10] Hayat, T., Shehzad, S. A., Qasim, M., and Obaidat, S. Radiative flow of Jeffrey fluid in a porous medium with power law heat flux and heat source. Nuclear Engineering and Design, 243, 15-19 (2012)

[11] Hayat, T., Shehzad, S. A., Qasim, M., and Obaidat, S. Thermal radiation effects on the mixed convection stagnation-point flow in a Jeffrey fluid. Z. Naturforsch., 66a, 606-614 (2011)

[12] Hayat,T.,Khan,M.,Siddiqui,A.M.,andAsghar.s.,Communications in Nonlinear Science and Numerical Simulation, 2007,12,910-919.

[13] Hayat.T, Umar Qureshi,M., Q.Hussain.Q.,Applied Mathematical Modelling 2009, 33,pp.1862-1873.

[14] Jamil, M., Khan, N. A., and Rauf, A. Oscillating flows of fractionalized second grade fluid. Mathematical Physics, 2012, 1-23 (2012) DOI 10.5402/2012/908386

[15] Kapur,J.N.,Mathematical Models in Biology and Medicine,1985,Affiliated East - west press Pvt.Lts,New 
Heat and Mass Transfer Effect on Peristalsis of Jeffrey Fluid in a Vertical Channel with Thermal Radiation and Heat Sources

York.

[16] Kay, W. M. Convective Heat and Mass Transfer, McGraw-Hill, New York (1966)

[17] Kothandapani, M. and Srinivas, S. Peristaltic transport of a Jeffrey fluid under the effect of magnetic field in an asymmetric channel. International Journal of Non-Linear Mechanics, 43, 915-924 (2008)

[18] Krishna Kumari S.V.H.N., Ramana Murthy P., Chenna Krishna Reddy, Ravi Kumar Y.V.K. M., Advances in Applied Sci.Res.,2011,2(2),428 - 436.

[19] Krishna Kumari. S. V. H. N. P, Ravi Kumar Y. V. K., Ramana Murthy M. V., Sreenadh S.: Peristaltic Pumping of a Conducting Jeffrey Fluid in a Vertical Porous Channel with Heat Transfer, Advances in Applied Science Research, Vol.2 (6), pp.439-453 (2011), Available online atwww.pelagiare searchlibrary. com

[20] Latham, T.W.,1966, Fluid motion in a peristaltic pump. M.SC, Thesis. Cambridge, Mass: MIT-Press.

[21] Liao, S. J. Beyond Perturbation: Introduction to Homotopy Analysis Method, Chapman and Hall, CRC Press, Boca Raton (2003)

[22] Mahmoud, M. A. A. and Waheed, S. E. Variable fluid properties and thermal radiation effects on flow and heat transfer in micropolar fluid film past moving permeable infinite flat plate with slip velocity. Applied Mathematics and Mechanics (English Edition), 33, 663-678 (2012) DOI 10.1007/s10483-012-1578-X

[23] Mekheimer, Kh.S. and Abd Elmaboud, Y.,Phys. Lett. A,2008, 372,1657-1665.

[24] Motsa, S. S., Hayat, T., and Aldossary, O. M. MHD flow of upper-convected Maxwell fluid over porous stretching sheet using successive Taylor series linearization method. Applied Mathematics and Mechanics (English Edition), 33, 975-990 (2012) DOI 10.1007/s10483-012-1599-X

[25] Nadeem, S. and Akbar, N. S. Peristaltic flow of a Jeffrey fluid with variable viscosity in an asymmetric channel. Z. Naturforsch., 64a, 713-722 (2009)

[26] Pal, D. and Mondal, H. The influence of thermal radiation on hydromagnetic Darcy-Forchheimer mixed convection flow past a stretching sheet embedded in a porous medium. Meccanica, 46, 739-753 (2011)

[27] Qasim, M., Hayat, T., and Obaidat, S. Radiation effect on the mixed convection flow of a viscoelastic fluid along an inclined stretching sheet. Z. Naturforsch., 67a, 195-200 (2012)

[28] Qi, H. and Jin, H. Unsteady helical flows of a generalized Oldroyd-B fluid with fractional derivative. Nonlinear Analysis: Real World Applications, 10, 2700-2708 (2009)

[29] Radhakrishnamacharya, G. and Srinivasulu,Ch., C.R. Mec.,2007,335,.369-373.

[30] Rashidi, M. M., Pour, S. A. M., and Abbasbandy, S. Analytic approximate solutions for heat transfer of a micropolar fluid through a porous medium with radiation. Communications in Nonlinear Science and Numerical Simulation, 16, 1874-1889 (2011)

[31] Ravi Kumar Y.V.K., Krishna Kumari S.V.H.N.P., Ramana Murthy M.V., Sreenadh S., Advances in Applied Sciences, 2011,2(3),396 - 406

[32] Shapiro, A.H., Jaffrin, M.Y and Weinberg, S.L., J. Fluid Mech, 1969. 37, 799-825.

[33] Shapiro,A.H., ,Proceedings of the Workshop in Ureteral Reflux in Children, 1967,109-126.

[34] Shit, G. C. and Halder, R. Thermal radiation and Hall effect on MHD flow, heat and mass transfer over an inclined permeable stretching sheet. Thermal Science, 15, S195-S204 (2011)

[35] Sud, V.K., Sekhon, G.S. and Mishra, R.K., Bull. Math. Biol,1977,. 39, no. 3,385-390.

[36] Vajravelu, K., Prasad, K. V., Sujatha, A., and Ng, C. O. MHD flow and mass transfer of chemically reactive upper convected Maxwell fluid past porous surface. Applied Mathematics and Mechanics (English Edition), 33, 899-910 (2012) DOI 10.1007/s10483-012-1593-8

[37] Vajravelu, K., HemadriReddy.R., Murugesan, M.,Int. Jr. of Fluid Mechanics Res.,2009,36, Issue 3, 244254.

[38] Wang, C. Y. The three-dimensional flow due to a stretching sheet. Physics of Fluids, 27, 1915-1917 (1984)

[39] Yin, F. and Fung, Y.C.,Trans. ASME J. Appl. Mech.,1969, 36, 579-58. 


\section{AUTHORS' BIOGRAPHY}

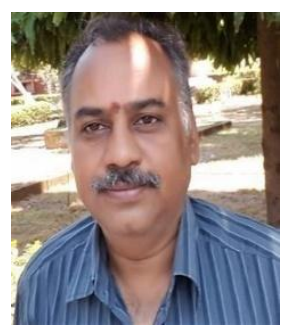

Dr. V. Ramesh Babu, a Professor, Department of Mathematics, Rashtriya Sanskrit Vidyapeetha, Tirupati, Chittoor (DT), A.P. He did his M.Sc. Applied Mathematics from S.V.University, in 1990 and Ph.D. degree in 2003 from S.V.University, Andhra Pradesh. He worked as lecturer from 1991 to 2009 in SV Arts College(TTD) and joined as Assistant Professor in 2009 in R.S.Vidyapeetha, Tirupati. He has published 11 National and International Journals and co-authored for two text books Numemerical Analysis and Integral Transforms both published by S.Chand \& Co New Delhi, He Edited two books one on proceedings of the 5 day National workshop on Ancient Indian Mathematics with a special focus on Vedic Mathematics sponsored by DST GOI and Leelavathi Ganitham both published by R.S.Vidyapeeth, Tirupati. He has conducted 6 workshops/seminars/conferences/DST projects and participated in different National and International Conferences. His areas of interest are Fluid Dynamics, Bio-mechanics, Heat and mass Transfer in Porous Medium, Chemical Reaction in Fluid Flows and Nano fluids

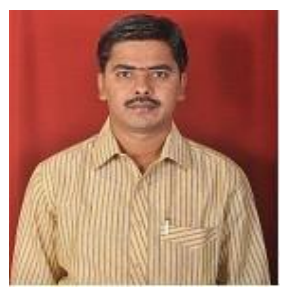

S. Sridhar is an Assistant Professor, Sree Venkateswara College of Engineering, North Rajupalem, SPSR NELLORE (DT). A.P. India since June 2014. He did his M.Sc. Mathematics from S.V.University, in 2007 and pursuing Ph.D. degree from Rayalaseema University, Kurnool, Andhra Pradesh from 2009. He has worked as Assistant Professor from different colleges since June 2007 to May 2014. He has participated in different National and International Conferences. His areas of interest are Fluid Dynamics and Bio-mechanics.

Citation: Sridhar, S \&V. Ramesh Babu(2019). Heat and Mass Transfer Effect on Peristalsis of Jeffrey Fluid in a Vertical Channel with Thermal Radiation and Heat Sources. International Journal of Scientific and Innovative Mathematical Research (IJSIMR), 7(2), pp.18-32. http://dx.doi.org/10.20431/2347-3142.0702003

Copyright:@ 2019Authors, Thisis an open-access article distributed under the terms of the Creative Commons Attribution License, which permits unrestricted use, distribution, and reproduction in any medium, provided the original author and source are credited. 